\title{
Theoretical and Experimental Evaluation of the Low $m / z$ Transmission of an Electrodynamic Ion Funnel
}

\author{
Jason S. Page, Aleksey V. Tolmachev, Keqi Tang, and Richard D. Smith \\ Biological Sciences Division, Pacific Northwest National Laboratory, Richland, Washington, USA
}

\begin{abstract}
The transmission of ions at low $\mathrm{m} / \mathrm{z}$ can often be either necessary for an application or problematic (e.g., when large numbers of low $\mathrm{m} / \mathrm{z}$ ions consume a large fraction of an ion trap's capacity). The low $\mathrm{m} / \mathrm{z}$ ion transmission limit of an electrodynamic ion funnel has been characterized using both experimental and theoretical approaches. A theoretical model is developed based on a series of infinite wire conductors that represent the ring electrodes of the ion funnel. Mathematical relationships for both low and high $\mathrm{m} / \mathrm{z}$ cutoffs of the idealized two-dimensional system are derived. The low $\mathrm{m} / \mathrm{z}$ cutoff is also evaluated through a series of experiments that show it is influenced by both the RF frequency and the DC electric field gradient. However, unlike multipole ion guides, there is no marked dependence of the low $\mathrm{m} / \mathrm{z}$ cutoff on the RF amplitude, in agreement with theoretical results. With this new understanding, ion funnels can be designed and configured to better match the $m / z$ range requirements for various applications. (J Am Soc Mass Spectrom 2006, 17, 586-592) (C 2006 American Society for Mass Spectrometry
\end{abstract}

$\mathrm{I}$ on transmission efficiency from the ion source to the mass spectrometer detector can be limited by many factors. For example, in electrospray ionizationmass spectrometry (ESI-MS), ions are initially created at atmospheric pressure and then transmitted through several stages of progressively lower pressure to the high vacuum of the mass analyzer. The combined ion losses can be substantial, with predictions of only one out of every $10^{2}-10^{4}$ ions generated by ESI at atmospheric pressure actually reaching the detector [1-3].

Considerable work has been done to improve the ion transmission by using ion guiding devices [4]. The most common ion guide is the radio frequency (RF)-only multipole that consists of 4,6 , or 8 rods in which a high RF voltage is applied (typically $1 \mathrm{MHz}$ at a few hundred $\mathrm{V}_{p-p}$ ). The RF voltage creates an effective potential well in the radial direction that confines the ions and allows for efficient ion transmission through the apertures between differential pumping stages and into the high vacuum region of the mass analyzer.

A variation of such multipole devices is the stacked ring ion guide (SRIG) [4-6], which consists of a series of flat washer-like rings arranged with small gaps between the electrodes, forming an ion tube or tunnel. Similar to multipole devices, an RF voltage is applied to each ring $180^{\circ}$ out of phase with respect to the neighboring rings, which creates an effective potential that

Published online February 28, 2006

Address reprint requests to Dr. Richard D. Smith, Biological Systems Analysis and Mass Spectrometry, Pacific Northwest National Laboratory, 3335 Q Avenue (K8-98), P.O. Box 999, Richland, WA 99352, USA.

confines the ion beam inside the SRIG and allows transmission through the device. Recently, a "travelingwave" SRIG has been implemented on an orthogonal time-of-flight (oTOF) mass spectrometer that uses a superimposed DC voltage pulse on the rings to carry the ions through the ion guide [7].

Expanding on the concept of a SRIG, we have previously developed a device called an ion funnel that replaces the traditional skimmer interface used in higher pressure ion sources, such as ESI, which improves the ion transmission efficiency in this region [8-11]. Our present ion funnel design consists of a stack of 100 ring electrode plates operating at a typical pressure range of 0.1-10 Torr. Each of the first $\sim 58$ ring electrodes has an inner dimension (i.d.) of $25.4 \mathrm{~mm}$; collectively, these electrodes create a traditional SRIG. The last $\sim 42$ ring electrodes decrease linearly in i.d., starting at $25.4 \mathrm{~mm}$ and ending at $2.5 \mathrm{~mm}$. A superimposed RF voltage and DC gradient is applied to the rings, which confines and transmits the ions through the device. This configuration allows ions entering the mass spectrometer to be captured, focused, and transmitted through a small aperture. Ion loss is greatly reduced or eliminated compared to a standard capillary-skimmer interface that only samples a fraction of the ions entrained in the gas jet exiting the inlet capillary.

Recent advances have further improved the performance of the ion funnel. For example, a disk electrode "jet disrupter" was placed in the entrance region of the ion funnel to disperse the gas jet exiting the heated inlet capillary, thereby reducing the gas load to the following stage and prohibiting neutrals from entering the mass spectrometer [12]. The jet disrupter has also been used 
as an "ion valve" to regulate the intensity of the ion beam for use with automated gain control to improve mass measurement accuracy [13]. In addition, a controllable $\mathrm{m} / \mathrm{z}$ filter was created at the exit of the ion funnel that removes lower mass chemical background species in some applications, and reducing down-stream space charge effects in ion trapping devices [14]. Ion funnels have also been designed with duel electrospray inlets that combine ion streams from multiple ion sources [15]. Additionally, ion funnels have been incorporated in ion mobility spectrometers to serve as ion traps/ gates and to eliminate ion diffusion loss in the drift tube, which greatly improves sensitivity [16]. The ion funnel has also been recently implemented in several Bruker Daltonics (Billerica, MA) ion source designs to reduce ion loss in atmospheric sources and to combine ESI and matrix-assisted laser desorption ionization (MALDI) into a single ion source [17].

The desire for improved sensitivity in a range of applications continues to expand the role of the ion funnel in mass spectrometry. It also emphasizes the need for a broader $\mathrm{m} / \mathrm{z}$ transmission range (especially for increased efficiency at low $\mathrm{m} / \mathrm{z}$ ) and the desirability of controlling the $\mathrm{m} / \mathrm{z}$ range transmitted. While the fundamental mechanisms of ion transmission and focusing properties of ion funnels have been broadly studied $[9,11,18,19]$, much of the work has centered on optimizing electrode configuration and spacing in conjunction with ion current, gas dynamic, and pressure effects. However, different published ion funnel configurations have demonstrated a variety of $\mathrm{m} / \mathrm{z}$ transmission ranges, and a detailed study of the $m / z$ dependence of ion transmission has not been reported.

Herein, we detail how the RF frequency and DC electric field gradient impact ion transmission. The $\mathrm{m} / \mathrm{z}$ transmission limit of the ion funnel is substantially independent of RF voltage (unlike traditional RF-only multipoles). We also present a theoretical model that explains the experimental results, along with mathematical relationships that relate the ion funnel $\mathrm{m} / \mathrm{z}$ transmission limit to the RF frequency and DC electric field.

\section{Experimental}

The ion funnel consisted of 100 ring electrodes made from $0.5 \mathrm{~mm}$ thick brass plates and separated by $0.5 \mathrm{~mm}$ thick Teflon spacers. The holes in the plates were made by wire electrical discharge machining (EDM) resulting in a flat inner surface. The tapered section of the ion funnel included 42 electrodes that linearly decreased in i.d., starting at $25.4 \mathrm{~mm}$ and ending at $2.5 \mathrm{~mm}$. These electrodes were followed by a DC only electrode (conductance limit) with an i.d. of $2.0 \mathrm{~mm}$. The $6.5 \mathrm{~mm}$ diameter jet disrupter electrode was located $\sim 2 \mathrm{~cm}$ from the exit of the inlet capillary. The circuitry was mounted onto two circuit boards and consisted of a chain of $500 \mathrm{k} \Omega$ resistors for the DC gradient and $10 \mathrm{nF}$ capacitors for the RF voltage and DC isolation. The boards were attached to the ion funnel with two custom (zero insertion force) ZIF connectors (Tactic Electronics, Plano, TX). The RF was applied to the ion funnel by a custom high-Q head driven by a waveform generator (model HP 33120A, Hewlett-Packard, Loveland, CO) and an RF power amplifier (model ENI 2100L, Electronic Navigation Industries, Rochester, NY). The DC voltages were supplied by a 9 output DC power supply (model TD-9500, Spectrum Solutions, Russellton, PA). The RF and DC voltages were varied throughout the experiments (values given in the Results section). The conductance limit DC voltage was maintained at $5 \mathrm{~V}$ less than the voltage on the last funnel electrode, and the potential on the heated inlet capillary was $10 \mathrm{~V}$ greater than the voltage on the first funnel electrode. The jet disrupter voltage was adjusted for maximum ion transmission which resulted in a voltage range of 15 to $25 \mathrm{~V}$ less than the first funnel electrode voltage, and the pressure in the ion funnel chamber was 1.9 torr measured by a "mini convectron" thermocouple gauge that was original to the mass spectrometer.

An Agilent MSD1100 single quadrupole mass spectrometer (Agilent Technologies, Santa Clara, CA) modified with an ESI/ion funnel source was used for the mass spectrometric analyses and is similar to a previously described system [15]. Ions were created at atmospheric pressure by ESI. ESI emitters were prepared by pulling sections of $100 \mu \mathrm{m}$ i.d./ $200 \mu \mathrm{m}$ o.d. fused silica capillary (Polymicro Technologies, Phoenix, AZ) with a Model 2000 capillary puller (Sutter Instrument Company, Novato, CA). The ESI emitter was connected to a transfer capillary and a $25 \mu \mathrm{L}$ syringe (Hamilton, Las Vegas, NV) by a stainless steel union, which also served as the connection point for the ESI voltage. All solutions were infused from a Harvard Apparatus Model 22 syringe pump (Holliston, MA) at a flow rate of 0.3 $\mu \mathrm{L} / \mathrm{min}$. Voltages from $1.4-1.5 \mathrm{kV}$ were applied to the ESI emitter via a Burtan high voltage power supply (Model 205B-03R, Hicksville, NY).

Mass spectra of a calibrant solution (Agilent Technologies, product no. G2421A), which contained a mixture of betaine and substituted triazatriphosphorines that produce singly charged ions by ESI over a broad mass range [20] were acquired in positive ESI mode with a $0.1 \mathrm{~m} / \mathrm{z}$ step size. Each spectrum used was the result of a 10 scan average to reduce the effects of any intensity fluctuations in the electrospray.

\section{Results and Discussion}

This study evaluated the effect of RF frequency upon ion funnel transmission efficiency using a single quadrupole mass spectrometer. In the first part of the study, a DC electric field gradient of $19.1 \mathrm{~V} / \mathrm{cm}$ was applied to the ion funnel and the RF frequency was varied while maintaining a RF peak-to-peak voltage $\left(\mathrm{V}_{p-p}\right)$ of $80 \mathrm{~V}$. Figure $1 \mathrm{a}$ and $\mathrm{b}$ show mass spectra acquired at $\mathrm{RF}$ frequencies of 700 and $300 \mathrm{kHz}$, respectively. Reducing the frequency increased the cutoff for low $\mathrm{m} / \mathrm{z}$ ions; 

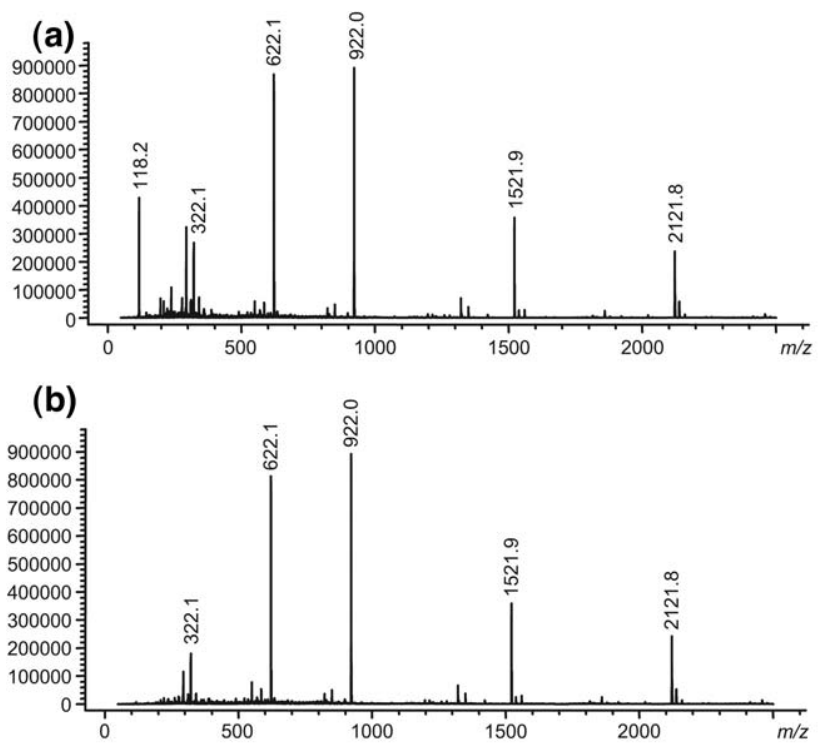

(c)

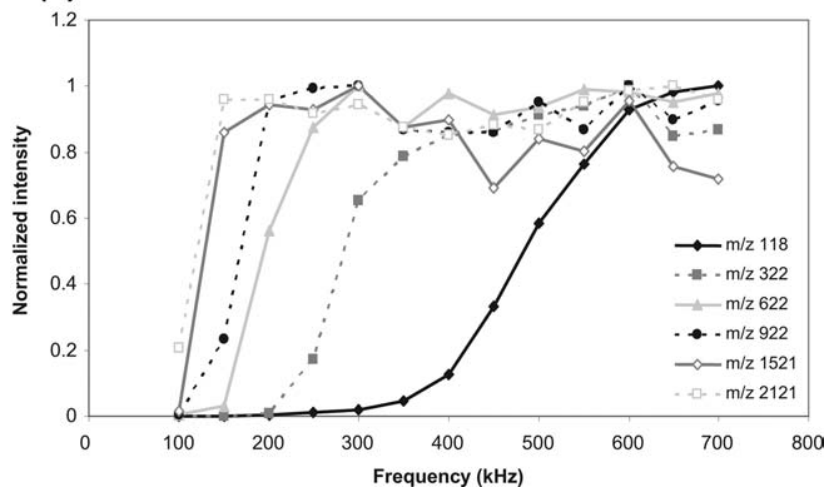

Figure 1. Mass spectra showing the effect of ion funnel RF frequency on the intensity of six calibrant peaks. These mass spectra were acquired using a $700 \mathrm{kHz}$ (a) and $300 \mathrm{kHz}$ (b) RF frequency. The effect of the RF frequency on the low $\mathrm{m} / \mathrm{z}$ cutoff of the ion funnel is shown in the bottom plot (c) of normalized intensity versus ion funnel RF frequency. For all data, the funnel $\mathrm{RF}$ amplitude was maintained at $80 \mathrm{~V}_{p-p}$, the DC gradient was 19.1 $\mathrm{V} / \mathrm{cm}$, and the pressure was 1.9 torr.

most noticeably, the $\mathrm{m} / \mathrm{z} 118.2$ peak was eliminated and the $m / z 322.1$ peak was reduced, while the intensity of the other four higher mass calibrant peaks remained unchanged. To better characterize this effect, the RF frequency was varied from 100 to $700 \mathrm{kHz}$. Mass spectra were acquired in $50 \mathrm{kHz}$ increments, and the normalized intensities of the six calibrant peaks were recorded and plotted in Figure 1c. At $100 \mathrm{kHz}$, the ions were not transmitted through the ion funnel (or poorly transmitted in the case of $m / z 2121.8$ ), corresponding to a low $\mathrm{m} / \mathrm{z}$ limit of $\sim 2100$. Increasing the RF frequency shifted the cutoff to lower $\mathrm{m} / \mathrm{z}$. The intensity of each calibrant peak increased initially as the RF frequency increased and eventually leveled off at a sufficiently high RF frequency. In other words, lower $\mathrm{m} / \mathrm{z}$ ions required higher RF frequencies for effective transmission through the ion funnel.

Previous work that investigated ion transmission properties of the ion funnel used computer simulations and theoretical models based on the exact dimensions and designs of the funnel $[11,18]$. Here, we propose a simplified approach that specifically addresses the $\mathrm{m} / \mathrm{z}$ range as a function of RF frequency. Consider a set of infinite line conductors that carry an alternate polarity voltage $V_{0}$, as illustrated in Figure 2a. This model has been used previously to estimate the stored ion capacity of an RF SRIG [21]. This two-dimensional (2-D) structure is expected to provide a rough approximation for ions positioned close to the inner ion funnel surface, with radial positions $r$ much greater than the ring electrode spacing $d$, as shown in Figure $2 \mathrm{~b}$.

The potential distribution for the 2-D system can be approximated as follows:

$$
V(h, z)=V_{0} \exp (-h / \delta) \sin (z / \delta)
$$

Here, the $h$ coordinate is perpendicular to the plane of wires, $h>0$, and $z$ is the coordinate along the plane, as shown in Figure 2a. The characteristic length $\delta$ is related to the distance between wires $d$ as $\delta=d / \pi$. The potential distribution in eq 1 delivers an exact solution to Laplace's equation with a boundary condition at $h=$

\section{(a)}

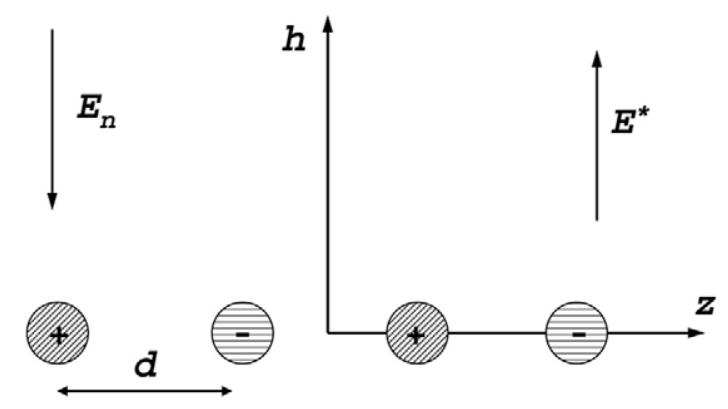

(b)

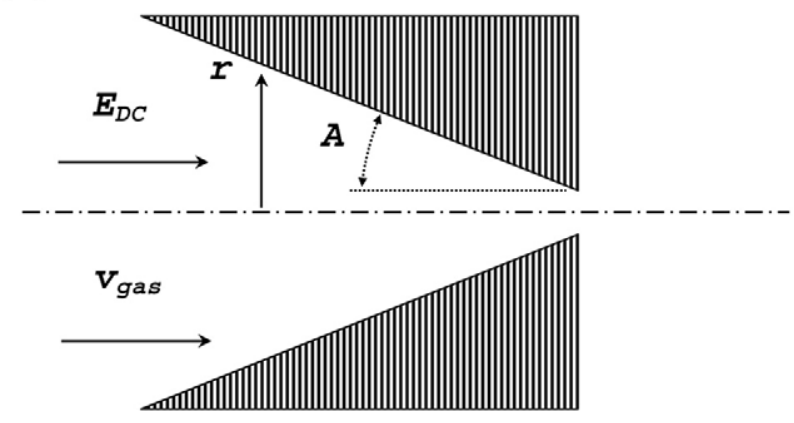

Figure 2. (a) A diagram of the infinite wire conductor model used to simulate the effect of RF and DC fields on the ion transmission in an ion funnel. The shaded circles represent infinite wires projected into the plane of the paper with the corresponding polarity. The DC electric field component normal to the inner surface of the ion funnel is represented by $E_{n}$ and the effective field by $E^{*}$ the coordinate system $h, z$ is shown. (b) A diagram explaining notations for the ion funnel parameters: $E_{D C}$ is the DC electric field intensity, $v_{\text {gas }}$ is the gas flow velocity, $r$ is the instantaneous radial coordinate of an ion and $\mathrm{A}$ is the ion funnel angle. 
$0: V(0, z)=V_{0} \sin (z / \delta)$. The 2-D wire arrangement does not exactly fit this boundary condition, but at a distance $h \gtrsim \delta$ the potential is still reasonably well approximated by eq 1 .

Applying the time-dependent RF potential $V_{0}(t)=$ $V_{R F} \sin (\omega t)$ [4] to the 2-D wire arrangement effectively focuses the ions, and the effective potential can be estimated using eq 1 as follows:

$$
V^{*}(h)=z e V_{R F}^{2} \exp (-2 h / \delta) / 4 m \omega^{2} \delta^{2}
$$

Here, $z$ is the ion charge state, $e$ is the elementary charge, and $\omega=2 \pi f$ is the angular frequency, where $f$ is the RF frequency applied to the ion funnel. The effective potential in eq 2 is expressed in volts for convenience. Note that more common relationships use joules as the effective potential units, resulting in proportionality to ze squared [4].

The DC gradient applied to the ion funnel ring electrodes produces a DC electric field that has a component normal to the inner surface, $E_{n}=E_{D C} \sin$ $A$. Here, $A$ is the ion funnel angle such that $\tan A=$ $\Delta D / 2 d=0.25$, where $\Delta D=0.5 \mathrm{~mm}$ is the i.d. difference of two neighboring ring electrodes. In our simplified arrangement, the normal component of the DC gradient can be accounted for by adding the normal field $E_{n}$ that drives ions towards the wire plane (Figure 2a).

For an ion funnel at $\sim 1$ torr gas pressure, ions experience multiple collisions with neutrals over a characteristic length $\sim d=1 \mathrm{~mm}$. The ion kinetic energy is rapidly dissipated in the collisions; therefore, the ion motion is governed by the field intensities and not by the local potential. The DC field $E_{n}$ forces ions toward the boundary formed by the RF electrodes. This force is counterbalanced by the effective focusing field $E^{*}=-\operatorname{grad}\left(V^{*}\right)$. The effective potential $V^{*}$ at elevated gas pressure should be corrected by a coefficient $\gamma$ to account for the effective potential decline due to ion-neutral collisions [22]. For a pressure of $\sim 1$ torr and a relevant frequency range of the ion funnel, the parameter can be estimated as $\sim 0.5<\gamma<1$. For a simple order of magnitude evaluation, we assume $\gamma \sim 1$, and arrive at the following expression to describe the effective field:

$$
E^{*}(h)=z e V_{R F}^{2} \exp (-2 h / \delta) / 2 m \omega^{2} \delta^{3}
$$

Ions are trapped at a potential well minimum, where the DC field $E_{n}$ is balanced by the effective field $E^{*}(h)$. Ions with higher $m / z$ are trapped closer to the grid, i.e., at smaller $h$ values. When positions are close enough, $h$ $=h_{0}$ and ion trajectories may intercept with the grid, which results in neutralization or ion loss. Assuming a balance of $E_{n}$ and $E^{*}\left(h_{0}\right)$, we arrive at the following estimation for the high $\mathrm{m} / \mathrm{z}$ limit of ions confined by the model 2-D structure:

$$
(m / z)_{\text {high }}=e V_{R F}^{2} \exp \left(-2 h_{0} / \delta\right) / 2 m_{u} \omega^{2} \delta^{3} E_{n}
$$

Here $m_{u}=1.6605^{*} 10^{-27} \mathrm{~kg}$ is the atomic mass unit. Note that the high $\mathrm{m} / \mathrm{z}$ value is very sensitive to the assumed position of ion loss $h_{0}$. The $h_{0}$ value depends on the grid configuration details and other parameters; for an order of magnitude estimation we chose $h_{0}=\delta$.

The low $\mathrm{m} / \mathrm{z}$ limit can be estimated by using the adiabaticity parameter $\eta$ [4]. The potential distribution eq 1 results in the following relationship for $\eta$ :

$$
\eta(h)=2 z e V_{R F} \exp (-h / \delta) / m \omega^{2} \delta^{2}
$$

The effective focusing is efficient if the adiabaticity parameter is smaller than a certain threshold value $\alpha \lesssim 1$ [4]:

$$
\eta(h)<\alpha
$$

This condition should hold at all $h$ positions down to the field balance, $E_{n}=E^{*}(h)$. Substitution of the condition into eq 3 using eqs 5 and 6 leads to the following low $\mathrm{m} / \mathrm{z}$ cut off relationship:

$$
(m / z)_{\mathrm{low}}=8 e E_{n} / m_{u} \omega^{2} \delta \alpha^{2}
$$

Thus, the model system of RF wires with an additional DC electric field acts as a broad band pass $\mathrm{m} / \mathrm{z}$ filter. The ions within the range given by eqs 4 and 7 are trapped in a potential well formed by a DC gradient from one side and the effective potential gradient from the other side. The adiabaticity condition $\eta<\alpha \sim 1$ is not exact, and one can expect that the low $\mathrm{m} / \mathrm{z}$ cutoff eq 7 will not be as sharp as for RF-only quadrupoles. Rather, the cutoff should be similar to the more gradual decrease in low $\mathrm{m} / \mathrm{z}$ ion transmission observed for RF multipoles, consistent with experimental observation (Figure 1c).

The mathematical relationships were tested using computer simulations of ion motion in the proposed model 2-D system. The ion trajectories were calculated for the fast changing RF potential that had a spatial distribution defined by eq 1 with an additional DC gradient corresponding to the normal DC electric field $E_{n}$. The ion-neutral interactions were accounted for using an algorithm developed previously for modeling the ion funnel $[11,18]$. The model confirmed that the field balance approach can be used for evaluation of the ion equilibrium position under gas pressure conditions of interest here $(\mathrm{p} \sim 1$ torr). From a first principles perspective, the point of field balance corresponds to the effective potential-well minimum. In vacuum conditions, ions oscillate around the potential minimum with macromotion amplitude corresponding to the oscillation energy [4]. At $\sim 1$ torr, an ion free path $L_{c}$ is small, $L_{c}<d\left(m_{g} / m\right)$, where $m_{g}$ is the gas molecule mass. In other words, the ion kinetic energy is dissipated over a small path compared to the characteristic scale $d$. This explains why the positions of ions trapped in the effective potential well at high-pressure are close to the 
potential well minimum, independent of the initial ion trajectories.

The above model shows that ions can be trapped in the potential well formed by the DC gradient on one side and the effective potential from the plane of RF wires on the other side. The trapping efficiency is reduced for low $\mathrm{m} / \mathrm{z}$ ions in agreement with eq 7 for $\alpha \approx 1$. The adiabaticity threshold $\alpha$ that corresponds to the decline of effective focusing is also dependent on the pressure and cross section assumed. We found it appropriate to use $\alpha \approx 1$ for the range of parameters in our study. The trapped ions perform a random diffusional motion around the potential well minimum defined by the field balance. As the ion mass approaches the high $\mathrm{m} / \mathrm{z}$ limit given by eq 4 , the potential well approaches the RF wires. Simulations were consistent with the above assumption for the ion neutralization position $h_{0} \approx \delta$, assuming that the grid wires have diameters $<\approx \delta$.

To test whether the low $\mathrm{m} / \mathrm{z}$ cutoff is dependent on the magnitude of the DC electric field (eq 7), experiments similar to those shown in Figure 1 were conducted with DC field gradients of 9.0 and $29.1 \mathrm{~V} / \mathrm{cm}$. Figure 3 shows plots of the intensity of $\mathrm{m} / \mathrm{z} 118.2$ versus RF frequency, illustrating the observed changes in the ion transmission efficiency due to the DC field; a decreased DC gradient shifts the cutoff frequency to a lower $\mathrm{m} / \mathrm{z}$.

A comparison of experiment and theory for the cutoff frequency versus $\mathrm{m} / \mathrm{z}$ for the three DC gradients is shown in Figure 4. Each point in the graph was taken from the intensity versus RF frequency graphs for each calibrant peak (i.e., the plot in Figure 3 was used for the $m / z 118.2$ points). The plotted cutoff point for the $\mathrm{m} / \mathrm{z}$ of each calibrant peak was determined by the frequency at which the intensity dropped by 50\% along with the theoretical predictions. The results show: (1) the DC gradient of the ion funnel has an effect on the ion transmission range and, as indicated by eq 7 , a lesser field decreases the RF frequency requirement for a particular $m / z$; (2) the mathematical relationship used to define the low $\mathrm{m} / \mathrm{z}$ cutoff provides good agreement with experiment (even though

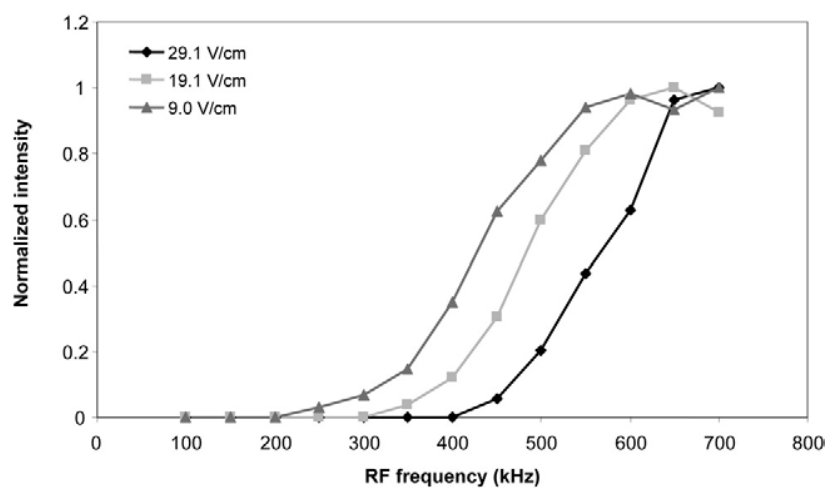

Figure 3. The effect of the RF frequency on the ion funnel transmission for $m / z 118.2$ as a function of three different DC voltage gradients. The RF amplitude was $80 \mathrm{~V}_{p-p}$ for all three datasets.

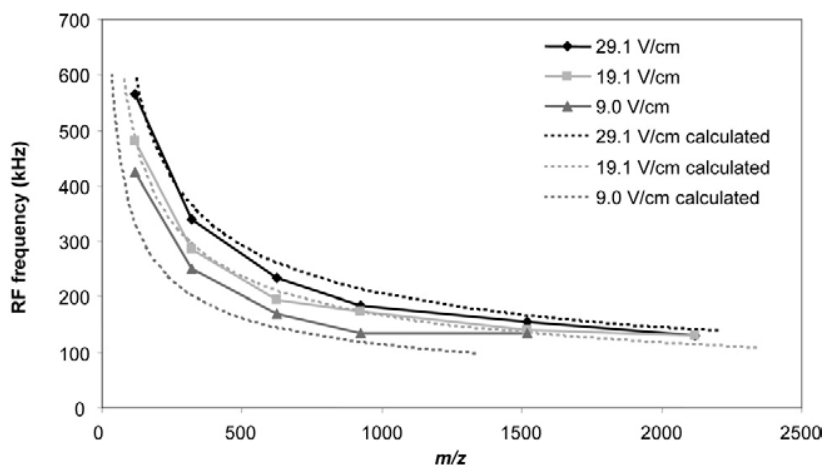

Figure 4. RF frequency versus the low $m / z$ cutoff of an ion funnel for three different DC voltage gradients. The data points were acquired using transmission versus RF frequency plots for each calibrant peak, similar to those for $m / z 118.2$ in Figure 3. The plotted RF frequency for the $\mathrm{m} / \mathrm{z}$ of each calibrant peak was determined by recording the frequency where the intensity was reduced to $50 \%$. The RF potential was $80 \mathrm{~V}_{p-p}$. The theoretical predictions are from eq 7 , using the ion funnel parameters from these experiments and $\alpha=1$.

it was built using an infinite line conductor approach); (3) both the 29.1 and $19.1 \mathrm{~V} / \mathrm{cm}$ DC fields follow the theoretical model, but the $9.0 \mathrm{~V} / \mathrm{cm}$ gradient deviates more significantly from prediction. The latter deviation is most likely due to the influences from the background gas, as discussed below.

It is of interest that eq 7 indicates the ion funnel RF voltage should not affect the low $\mathrm{m} / \mathrm{z}$ cutoff. To investigate this, we examined the $m / z$ cutoff at four different RF amplitudes, 60, 80, 100, and $120 \mathrm{~V}_{p-p}$ (Figure 5). The results show similar performance for all four datasets, and they align very well to both themselves and theoretical prediction, indicating that $R F$ voltage amplitudes have no significant effect on the $\mathrm{m} / \mathrm{z}$ cutoff over this range.

The relative independence of the RF amplitude on

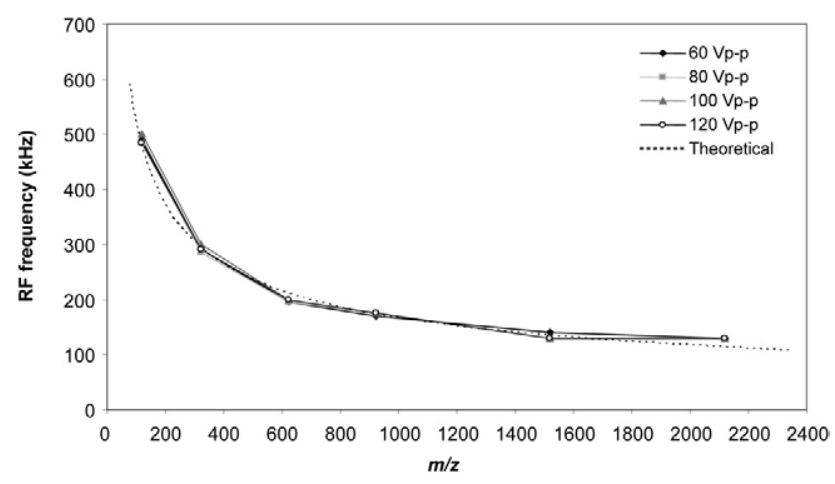

Figure 5. RF frequency versus the low $m / z$ cutoff of an ion funnel for four different RF amplitudes. The data points were acquired using transmission versus RF frequency plots for each calibrant peak, similar to those shown in Figure 3 for $m / z$ 118.2. The plotted RF frequency for the $m / z$ of each calibrant peak was determined by recording the frequency at which the intensity was reduced to $50 \%$. The DC field gradient was $19.1 \mathrm{~V} / \mathrm{cm}$ for all datasets. The theoretical line is a solution to eq 7, using the ion funnel parameters from these experiments and $\alpha=1$. 
the low $m / z$ cutoff is in apparent contrast to other RF focusing systems (e.g., quadrupole and octopole devices). This behavior can be explained by considering the 2-D structure (Figure 2a) using two different RF amplitudes applied to the wires, and ions with a $\mathrm{m} / \mathrm{z}$ that provide a solution to eq 7. The potential well minimum that corresponds to the field balance $E^{*}(h)=$ $-E_{n}$ will be shifted farther away from the grid as the RF amplitude increases, according to eq 3 . The shift of the balance position fully compensates the increased RF amplitude. Ions below the balance position cannot be stopped by the effective RF focusing since the adiabaticity condition, eq 6, does not hold, irrespective of the RF voltage applied. Increased RF amplitudes shift equilibrium further away from the grid so that the adiabaticity parameter near the point of equilibrium stays unchanged.

We conclude that our experimental results are generally consistent with the theoretical relationships derived for the simplified 2-D geometry. This consistency suggests that low $\mathrm{m} / \mathrm{z}$ ion losses occur at positions before the exit orifice where the condition of large radius, $r \gg d$, is applicable.

The drag force from the gas flow should also contribute to the ion loss. The drag force can be expressed in electric field units using the ion mobility coefficient K:

$$
K E_{\text {gas }}=v_{\text {gas }}
$$

Using an ion mobility value $K \sim 10^{3} \mathrm{~cm}^{2} / V s$ that is characteristic for 1 torr $\mathrm{N}_{2}$ and a gas flow velocity $v_{\text {gas }} \sim$ $100 \mathrm{~m} / \mathrm{s}$, we arrive at an order of magnitude estimation for the effective field created by the gas flow:

$$
E_{\mathrm{gas}} \sim 10 \mathrm{~V} / \mathrm{cm}
$$

It follows that the gas flow can contribute markedly to the low $m / z$ curves shown in Figure 4 . This contribution should be more pronounced for high $\mathrm{m} / \mathrm{z}$ ions because of lower mobility values and correspondingly higher $E_{\text {gas }}$ values, which possibly explains the suppressed dependence on $E_{D C}$ for high $\mathrm{m} / \mathrm{z}$ ions, as observed experimentally. In a similar fashion, the deviation from the theoretical curve in Figure 4 for $E_{D C}=9 V / \mathrm{cm}$ is due to the gas dynamic contribution being more pronounced for lower $E_{D C}$.

We did not characterize the high $\mathrm{m} / \mathrm{z}$ transmission behavior of the ion funnel in this experimental study since the 2-D model represented by eq 4 gives $(\mathrm{m} / \mathrm{z})_{\text {high }}$ $>10,000$ for the conditions used in our experiments. In practice, a decline in high $\mathrm{m} / \mathrm{z}$ transmission may be observed at a lower $\mathrm{m} / \mathrm{z}$ value due to the ion funnel exit geometry, pressure, space charge conditions, and acceptance of a multipole downstream of the ion funnel [23]. Further studies will address the high $\mathrm{m} / \mathrm{z}$ performance in the context of the new 2-D theoretical model.

\section{Conclusions}

The expanding role of the ion funnel in a variety of MS applications has increased the desirability of a broader $\mathrm{m} / \mathrm{z}$ ion funnel transmission range (especially for increased efficiency of low $\mathrm{m} / \mathrm{z}$ ), and for an improved understanding of ion losses. We have investigated properties of the ion funnel that affect the transmission efficiency and have developed a theoretical understanding and mathematical relationships that model the ion transmission properties.

We have discovered that the ion funnel can be tuned for improved low $\mathrm{m} / \mathrm{z}$ transmission by increasing the RF frequency and decreasing the DC field gradient, and that the low $\mathrm{m} / \mathrm{z}$ cutoff is not affected by the amplitude of the RF voltage, in contrast to conventional multipole ion guides that operate at lower pressures. Additionally, the simple mathematical model developed based upon infinite wire conductors fits the experimental data reasonably well and provides solutions for the ion funnel transmission limits. We believe that this new understanding will aid the design of ion funnels to better match $\mathrm{m} / \mathrm{z}$ transmission requirements for specific applications.

\section{Acknowledgments}

Portions of this research were supported by the U.S. Department of Energy (DOE) Office of Biological and Environmental Research and the NIH National Center for Research Resources (grant RR 18522). Experimental work was performed in the Environmental Molecular Sciences Laboratory, a DOE national scientific user facility at the Pacific Northwest National Laboratory (PNNL) in Richland, Washington. PNNL is operated by Battelle for the DOE under contract no. DE-AC05-76RLO 1830.

\section{References}

1. Cech, N. B.; Enke, C. G. Practical Implementations of Some Recent Studies in Electrospray Ionization Fundamentals. Mass Spectrom. Rev. 2001, 20, 362-387.

2. Kebarle, P.; Tang, L. From Ions in Solution to Ions in the Gas Phase. Anal. Chem. 1993, 65, 972A-986A.

3. Smith, R. D.; Loo, L. A.; Edmonds, C. G.; Barinaga, C. J.; Udseth, H. R. New Developments in Biochemical Mass Spectrometry Electrospray Ionization. Anal. Chem. 1990, 62, 882-899.

4. Gerlich, D. In State-Selected and State-to-State Ion Molecule Reaction Dynamics, Vol. LXXXII, Part 1. Baer, M.; Ed., John Wiley: New York, NY, 1992; pp 1-176.

5. Bahr, R.; Gerlich, D.; Teloy, E. Verhandl. Dtsch. Phys. Ges. 1969, 4, 343.

6. Luca, A.; Sclemmer, S.; Cermak, I.; Gerlich, D. On the Combination of a Linear Field Free Trap with a Time-of-Flight Mass Spectrometer. Rev. Sci. Instrum. 2001, 72, 2900-2908.

7. Giles, K.; Pringle, S. D.; Worthington, K. R.; Little, D.; Wildgoose, J. L.; Bateman, R. H. Applications of a Traveling Wave-Based Radio-FrequencyOnly Stacked Ring Ion Guide. Rapid Commun. Mass Spectrom. 2004, 18, 2401-2414

8. Taeman, K.; Tolmachev, A. V.; Harkewicz, R.; Prior, D. C.; Anderson, G.; Udseth, H. R.; Smith, R. D.; Bailey, T. H.; Rakov, S.; Futrell, J. H. Design and Implementation of a New Electrodynamic Ion Funnel. Anal. Chem. 2000, 72, 2247-2255.

9. Shaffer, S. A.; Tang, K.; Anderson, G. A.; Prior, D. C.; Udseth, H. R.; Smith, R. D. A Novel Ion Funnel for Focusing Ions at Elevated Pressure Using Electrospray Ionization Mass Spectrometry. Rapid Commun. Mass Spectrom. 1997, 11, 1813-1817.

10. Shaffer, S. A.; Prior, D. C.; Anderson, G. A.; Udseth, H. R.; Smith, R. D. An Ion Funnel Interface for Improved Ion Focusing and Sensitivity Using Electrospray Ionization Mass Spectrometry. Anal. Chem. 1998, 70, 4111-4119.

11. Shaffer, S. A.; Tolmachev, A. V.; Prior, D. C.; Anderson, G. A.; Udseth, H. R.; Smith, R. D. Characterization of an Improved Electrodynamic Ion 
Funnel Interface for Electrospray Ionization Mass Spectrometry. Anal. Chem. 1999, 71, 2957-2964.

12. Kim, T.; Tang, K.; Udseth, H. R.; Smith, R. D. A Multicapillary Inlet Jet Disruption Electrodynamic Ion Funnel Interface for Improved Sensitivity Using Atmospheric Pressure Ion Sources. Anal. Chem. 2001, 73, 4162-4170.

13. Page, J. S.; Bogdanov, B.; Vilkov, A. N.; Prior, D. C.; Buschbach, M. A.; Tang, K.; Smith, R. D. Automatic Gain Control in Mass Spectrometry Using a Jet Disrupter in an Electrodynamic Ion Funnel. J. Am. Soc. Mass Spectrom. 2005, 76, 244-253.

14. Page, J. S.; Tolmachev, A. V.; Tang, K.; Smith, R. D. Variable Low-Mass Filtering Using an Electrodynamic Ion Funnel. J. Mass Spectrom. 2005, 40, 1215-1222.

15. Tang, K.; Tolmachev, A. V.; Nikolaev E.; Zhang, R.; Belov, M. E.; Udseth, H. R.; Smith, R. D. Independent Control of Ion Transmission in a Jet Disrupter Dual-Channel Ion Funnel Electrospray Ionization MS Interface. Anal. Chem. 2002, 74, 5431-5437.

16. Tang, K.; Shvartsburg, A. A.; Lee, H. N.; Prior, D. C.; Buschbach, M. A.; Li, F. M.; Tolmachev, A. V.; Anderson, G. A.; Smith, R. D. HighSensitivity Ion Mobility Spectrometry/Mass Spectrometry Using Electrodynamic Ion Funnel Interfaces. Anal. Chem. 2005, 77, 3330-3339.

17. Stacey, C.; Kim, T.; Zhang, Z.; Knudsen, T.; Park, M. Simultaneous Accurate Mass ESI and MALDI Spectral Acquisition with a New Ion
Source. Proceedings of the 53rd ASMS Conference on Mass Spectrometry; San Antonio, TX, June, 2005; MP245.

18. Tolmachev, A. V.; Kim, T.; Udseth, H. R.; Smith, R. D.; Bailey, T. B.; Futrell, J. H. Simulation-Based Optimization of the Electrospray Ion Funnel for High Sensitivity Electrospray Ionization Mass Spectrometry. Int. J. Mass Spectrom. 2000, 203, 31-47.

19. Lynn, E. C.; Chung, M.-C.; Han, C.-C. Characterizing the Transmission Properties of an Ion Funnel. Rapid Commun. Mass Spectrom. 2000, 14, 2129-2134.

20. Flanagan,, J. M. Mass Spectrometry Calibration Using Homogeneously Substituted Fluorinated Triazatriphosphorines. U.S. Patent 1999, $5,872,357$.

21. Tolmachev, A.; Udseth, H. R.; Smith, R. D. Charge Capacity Limitations of Radio Frequency Ion Guides in Their Use for Improved Ion Accumulation and Trapping in Mass Spectrometry. Anal. Chem. 2000, 72, 970-978.

22. Tolmachev, A. V.; Chernushevich, I. V.; Dodonov, A. F.; Standing, K. G. A Collisional Focusing Ion Guide for Coupling an Atmospheric Pressure Ion Source to a Mass Spectrometer. Nucl. Instrum. Methods Phys. Res. B. 1997, 124, 112-119.

23. Tolmachev, A. V.; Kim, T.; Masselon, C. D.; Rakov, S. V.; Pasa-Tolic, L.; Harkewicz, R.; Tang, K.; Udseth, H. R.; Smith, R. D. Studies of the Ion Funnel Performance as a Function of Exit Geometries. Proceedings of the 49th ASMS Conference on Mass Spectrometry; Chicago, IL, May, 2001. 